\title{
INVESTIGACIÓN E INNOVACIÓN RESPONSABLES Retos teóricos y políticos
}

\author{
Andoni Eizagirre \\ Mondragon Unibertsitatea, Gipuzkoa, España
}

Resumen El artículo valora las nuevas vías para conceptualizar las relaciones entre ciencia, tecnología, políticas públicas y sociedad. Los Estudios de Ciencia y Tecnología anticipan modelos alternativos para comprender la gobernanza de la ciencia. Por su parte, en el ámbito institucional en el seno de la estrategia "Horizonte 2020" adquiere relevancia el lenguaje político asociado a la investigación y la innovación responsables (IIR) y se reclama que las actividades de investigación e ingeniería deben alinear mejor su proceso y resultados con los valores, las necesidades y las expectativas de la sociedad europea. El artículo establece un diálogo crítico entre los avances teóricos y el nuevo lenguaje político y sugiere que el significado, alcance y aplicación de un enfoque IIR dependerá de las dinámicas económicas y sociopolíticas por las cuales se instrumentalizan y regulan la ciencia y la tecnología.

Palabras-clave: investigación e innovación responsables, gobernanza, ciencia, sociedad.

Resumo O presente artigo valoriza as novas vias para conceber as relações entre ciência, tecnologia, política pública e sociedade. Os Estudos de Ciência e Tecnologia antecipam modelos alternativos para compreender a governança da ciência. Por seu lado, no âmbito institucional no seio da estratégia "Horizonte 2020" adquire relevância a linguagem política associada à investigação e inovação responsáveis (IIR) e defende-se que as atividades de investigação e engenharia devem alinhar melhor o seu processo e resultados com os valores, as necessidades e as expectativas da sociedade europeia. O artigo estabelece um diálogo crítico entre os avanços teóricos e a nova linguagem política e sugere que o significado, alcance e aplicação dum enfoque IIR dependerá das dinâmicas económicas e sociopolíticas pelas quais se instrumentalizam e regulam a ciência e a tecnologia.

Palavras-chave: investigação e inovação responsáveis, governança, ciência, sociedade.

Abstract The present article postulates the value of the new ways of looking at the relations between science, technology, public / policies and society. On the one hand, Science and Technology Studies / foreshadow / alternative models for understanding the governance of science. On the other, within the institutional framework at the heart of the "Horizon 2020" strategy, the political language associated with responsible research and innovation (RRI) is gaining in importance, arguing that those who engage in research and engineering activities should do a better job of aligning their processes and outcomes with the values, needs and expectations of European society. The article offers a critical dialogue between the theoretical advances and the new political language, and suggests that the significance, scope and application of an RRI-based focus will depend on the economic and sociopolitical dynamics by which science and technology are instrumentalised and regulated.

Keywords: responsible research and innovation, governance, science, society.

Résumé Cet article aborde les nouvelles voies pour concevoir les relations entre science, technologie, politique publique et société. Les Études de Science et Technologie proposent des modèles alternatifs pour comprendre la gouvernance de la science. Pour sa part, dans le cadre institutionnel de la stratégie "Horizon 2020", le langage politique associé à la recherche et à l'innovation responsables (RRI) prend de l'importance, en défendant que les activités de recherche et d'innovation doivent aligner davantage leurs processus et leurs résultats sur les valeurs, les besoins et les attentes de la société européenne. L'article établit un dialogue critique entre les avancées théoriques et le nouveau langage politique et suggère que le sens, la portée et l'application RRI dépendront des dynamiques économiques et sociopolitiques par lesquelles la science et la technologie sont instrumentalisées et régulées.

Mots-clés: recherche et innovation responsables, gouvernance, science, société. 


\section{Introducción}

En los últimos años las relaciones de la ciencia y la tecnología con su entorno se han ampliado y diversificado de manera extraordinaria. ${ }^{1}$ A este hecho se une la consolidación de nuevas dinámicas del conocimiento regidas por criterios prácticos, orientadas por el canon de la multidisciplinariedad, validadas crecientemente por racionalidades diversas y con una mayor sensibilidad hacia las expectativas socioeconómicas. Otro elemento distintivo es el referido a la emergencia de percepciones sociales críticas y exigentes ante un régimen de innovación que de manera creciente en los procesos de diseño, producción y comercialización se guía por la rentabilidad de mercado y que subestima sus efectos adversos y secundarios.

Esta serie de preocupaciones se detecta paralelamente en los ámbitos académico y político. Los Estudios de Ciencia y Tecnología sugieren nuevas vías para conceptualizar las relaciones entre ciencia, tecnología, políticas públicas y sociedad. Como bien sintetiza Irwin (2008), esta tradición de investigación, que en sus orígenes en las décadas de 1960 y 1970 desplaza el eje de análisis sociológico y etnográfico al estudio de los mecanismos de producción, uso y validación del conocimiento, últimamente amplía sus herramientas analíticas y conceptuales al ámbito de la política y aboga por comprender la gobernanza de la ciencia como resultado de una dinámica flexible, contextual y multidimensional en el que concurren una heterogeneidad de demandas sociales y económicas, intereses políticos, capacidades técnicas y factores socioculturales.

Por otra parte, los cambios también se observan en los ámbitos político e institucional. Apreciamos que en el lenguaje político se reconocen la importancia de los retos globales y las limitaciones socioéticas del régimen tecno-económico de la innovación, y por el cual los diferentes gobiernos nacionales y regionales se interesan paulatinamente de aspectos tradicionalmente omitidos en los sistemas de innovación. Un ejemplo lo encontramos en el emergente concepto de investigación e innovación responsables (IIR), elemento clave del 8.․ Programa Marco de I+D de la Unión Europea, "Horizonte 2020", que viene a confirmar una evolución que se inicia con el 7.ํㅜ Programa Europeo. El nuevo Programa Marco Europeo (2014-2020) reclama que las actividades de investigación e ingeniería deben alinear mejor su proceso y resultados con los valores, las necesidades y las expectativas de la sociedad europea. En este sentido, también las políticas nacionales de I+D precisan la importancia de abordar las necesidades sociales, las cuestiones éticas y los retos globales en la investigación y la innovación.

En este artículo establecemos un diálogo entre la tradición académica señalada y el nuevo discurso político que se reconoce en las instituciones europeas y nacionales. Esta tarea permite valorar críticamente las respectivas propuestas, e identifica sus avances y problemas, en la resolución de una controversia de carácter simultáneamente epistemológico, político, socioeconómico y ético. Es más, el concepto de IIR

1 La elaboración de este artículo ha contado con el apoyo del Departamento de Educación, Política Lingüística y Cultura del Gobierno Vasco (IT644-13). 
sugiere que la verdad y los códigos de la ciencia no pueden trasladarse de manera automática y lineal a la esfera política, lo que abre nuevos desafíos a las ciencias sociales y las humanidades, en aras a promover una discusión sobre el tipo de ciencia y tecnología que consideramos socialmente justo, adecuado y deseable.

El artículo se estructura como sigue: identificamos la tradición demarcacionista sobre hechos y valores en los estudios sociales de la ciencia y sus implicaciones en la comprensión reduccionista de las política de I+D; nos comprometemos con los Estudios de Ciencia y Tecnología y singularmente con el rol analítico de la perspectiva contextual, lo que nos permite una comprensión alternativa de la ciencia y su gobierno; proponemos los retos que apunta un enfoque IIR; anticipamos los desafíos que este nuevo contexto plantea a los Estudios de Ciencia y Tecnología y sus relaciones con un enfoque IIR; terminamos con una revisión del artículo y algunas consideraciones finales.

\section{Una comprensión reduccionista de las políticas de I+D}

Los análisis de las políticas de I+D han estado condicionados por los supuestos teóricos, analíticos y normativos que determinaron la comprensión del conocimiento científico. Debido a ello, asumen implícitamente buena parte de los presupuestos referidos a la causalidad y la imagen corriente de la ciencia, y en su versión triunfalista, asocian automáticamente la innovación científico-técnica y el progreso social.

De esta manera, en la fase de consolidación de las políticas de I+D también se presuponía que la producción, validación y justificación del conocimiento adquiría una consideración estrictamente interna al propio ámbito de la ciencia. Así, las formas de expresión más canónicas en el análisis de las políticas de I+D han sido, en la vertiente de su promoción, el análisis estadístico y la construcción de indicadores de la actividad científico-tecnológica (Godin, 2010), y, en la más reciente vertiente de su regulación, los análisis del riesgo (Shrader-Frechette, 1985; 1991). En este sentido, toda la reciente discusión ligada a la innovación y los indicadores de medición se reduce a la optimización de recursos y a la organización del sistema para mejorar la competitividad (Barben, 2007; Jamison, 2012). De la misma manera, toda evaluación de los resultados se basa en instrumentos técnicos y métricos. En este contexto de neutralidad axiológica de la ciencia y la tecnología, la reflexión sobre valores ha quedado relegada al ámbito externo y sujeto a la valoración ex post (Echeverría, 2002).

En relación al gobierno de la ciencia ha prevalecido una concepción demarcacionista, que concede al conocimiento un estatuto de objetividad, presupone un conocimiento indiscutible, seguro y útil, y así describe el escrutinio público como innecesario (Joly, Marris y Marcant, 2001). Es lo que certificamos en el registro de las corrientes positivistas o incluso en el más reciente de la "Ciencia y Sociedad" (Science and Society) a nivel europeo. Así, en el comienzo de su reformulación (EC, 2002), la gobernanza de la ciencia, en las iniciativas europeas, se orientó en la consideración de un fenómeno esencialmente ético, caracterizado como la gestión prudente y legitimadora de la actividad tecno-científica, diferenciado así de la ciencia 
en términos funcionales y desarrollado por reguladores externos (Felt, 2007; Irwin, 2006). Aquel giro social se articula todavía bajo criterios demarcacionistas (ciencia y política, conocimiento y ética, científico y regulador) que presupone un concepto de ciencia unidimensional, visible, libre de juicios de valor, a la vez que una traslación de la verdad (el código de la ciencia) a la racionalidad política (Liberatore, 2001). El nuevo interés por la sociedad se traducía así en iniciativas como la alfabetización en ciencia, la promoción del interés y la cultura científica, y la aplicación prudente del conocimiento producido y validado (Eizagirre, 2009).

Sin embargo, esa imagen ha sido sometida a diversas líneas de crítica. Los Estudios de Ciencia y Tecnología han superado las investigaciones demarcacionistas y han mostrado concluyentemente la multidimensionalidad de la ciencia, la funcionalidad no-externa de los valores e intereses, así como el carácter co-producido del orden científico y social (Irwin, 2008; Jasanoff, 2004; Mitcham, 2005). Se explica que lo que se entiende por conocimiento genuino, pertinente, robusto y relevante es más complejo e interactivo (Berkhout, Leach y Scoones, 2004; Callon, Lascoumes y Barthe, 2010), como exponemos en el siguiente apartado.

En consecuencia, por una parte, los análisis sobre investigación e innovación científico-tecnológica desmienten el empuje de la ciencia como motor de crecimiento y bienestar, ponen de manifiesto la naturaleza compleja de un proceso en el que se imbrican la ciencia, la política y la sociedad, y recientemente incluso se reconoce la necesidad de dirigirlo para responder a los retos globales de nuestras sociedades (OECD, 2012). Sea como fuere, la naturaleza de la ciencia y la tecnología, y las relaciones de estas entre sí y con la sociedad, tiene un carácter contingente, resultado de la interacción de los distintos actores en lucha por promover sus intereses. Por otra parte, se ha confirmado que los instrumentos analíticos y las bases legales en los procesos de regulación típicamente asociados al análisis del riesgo proceden a reducir aquello que no resulta cognoscible (incertidumbre, ignorancia, indeterminación) a través de asunciones de objetividad y controlabilidad que confieren legitimación a las instituciones científicas y políticas. Además, el conocimiento validado, certificado y utilizado como objetivo y seguro responde al modo como se apropian las demandas concurrentes en la sociedad (Wynne, 2002).

\section{Avances en la comprensión de la gobernanza de la ciencia}

En este apartado sostenemos que los Estudios de Ciencia y Tecnología han reformulado en la última década los problemas ligados a la actividad científico-tecnológica y su gobernanza. Singularmente, las políticas del conocimiento se asocian a concepciones de investigación e innovación inclusivas, a la producción, validación y evaluación bajo criterios robustos, y a valores más ligados a las preocupaciones, necesidades y expectativas de sus sociedades (Adger y Jordan, 2009; Ozolina et al., 2012). Destaca también que en los últimos años se han revisado los discursos y los planes de acción de la Unión Europea sobre las relaciones de la actividad científico-tecnológica con la sociedad (Anichini y de Cheveigné, 2012). Las instituciones sustraen lecciones de experiencias fallidas 
(innovaciones económicamente viables pero cuestionadas socialmente) y hay una mayor sensibilidad hacia futuros sociotécnicos colectivamente negociados. En el ámbito de la Unión Europea, el conflicto en torno a la biotecnología agroalimentaria ha supuesto un punto de inflexión.

Así, para los propósitos de nuestra investigación, aunque sea brevemente, queremos retener tres principales resultados, referidos al ámbito social e institucional de la ciencia y su relación con la sociedad. Estas sugerencias nos parecen relevantes, antes de pasar a analizar propiamente el enfoque IIR.

\section{El carácter sociotécnico de la innovación}

En primer lugar, queremos precisar el carácter sociotécnico de la innovación. En las últimas décadas, los programas de estímulo de la investigación y el desarrollo tecnológico reconocen los problemas del modelo lineal de innovación y proyectan una versión más compleja de los sistemas de investigación (Godin, 2010; OECD, 2010). Sin embargo, su carácter sociopolítico y los indicadores diseñados ante la emergencia de una nueva modalidad de actividad científica como respuesta al contexto de economía global responden exclusivamente a la optimización de recursos y las consiguientes transformaciones en la organización del sistema de ciencia y tecnología, orientado por la productividad y el crecimiento económico. Los cambios en la práctica de las actividades científico-tecnológicas y su organización siguen omitiendo la discusión sobre los aspectos ligados a los objetivos y las necesidades sociales y ambientales (Eizagirre, 2015; Miettinen, 2013; Bozeman y Sarewitz, 2011; van den Hove et al., 2012).

Además, en los Estudios de Ciencia y Tecnología se ha acentuado el interés por las propiedades que integra la nueva innovación tecno-económica (Felt, 2007). Así, a efectos analíticos, distinguimos, por una parte, aquellas contribuciones más orientadas a la crítica de la innovación tecno-económica. Estos análisis alertan de la inflación de las promesas y los futuribles, que juegan un rol determinante en la formación y estímulo del cambio científico y tecnológico, en tanto que las abstracciones orientadas al futuro guían actividades, proporcionan estructuras y legitimación, atraen interés y fomentan inversión. De hecho, este futuro prometido, que condiciona el desarrollo óptimo de la actividad investigadora, re-introduce el modelo lineal de innovación, en el que ideas brillantes se traducen en resultados predecibles y sujetos a gestión.

La crítica, por tanto, alerta de la paradoja del nuevo contexto social de la actividad científico-tecnológica: a la vez que se reconoce la naturaleza compleja de la innovación, se detecta que las propiedades normativas de la trayectoria sociotecnológica están instrumentalizadas para resultados macroeconómicos (Marklund, Vonortas y Wessner, 2009). En otras palabras, el proceso multidireccional de variación y selección que consolidan las formas científico-tecnológicas se rigen mayormente por la utilidad y la rentabilidad de mercado. Otras perspectivas más recientes se preguntan por la interrelación entre la ciencia, su organización y la regulación neoliberal de las economías capitalistas, entre las que destacan las tesis sobre "convergencia asimétrica" (Kleinman y Vallas, 2001), "régimen de privatización globalizada" (Mirowski y 
Sent, 2008) y "ciencia neoliberal" (Slaughter y Rhoades, 2004; Canaan y Shumar, 2008).

No obstante, junto a estas advertencias críticas sobre las trayectorias de la innovación tecnológica, tradicionalmente ignoradas en las ciencias económicas, por otra parte, encontramos aquellos estudios dirigidos a enriquecer la innovación sociotécnica, las políticas de investigación e innovación responsables, la interdisciplinariedad en las prácticas científico-tecnológicas, la gobernanza anticipatoria, la pluralización de criterios en el diseño y la validación, etc. (Bolay et al., 2012; Leach, Scoones y Stirling, 2010).

\section{Ciencia, riesgo y valores}

En segundo lugar, nos parece importante enfatizar la función de los valores (epistemológicos, normativos, pragmáticos, éticos) en la regulación de la actividad científico-tecnológica y sus impactos adversos (Shrader-Frechette, 2011). Las teorías de decisión sobre el riesgo manejan un concepto probabilista y previsible del efecto adverso, que ha posibilitado heredar la vasta cultura epistemológica que estima todo como magnitud objetiva, abandonada a la ilusión de la medición y la calculabilidad (Eizagirre, 2013a; Lane, Klauser y Kearnes, 2012; Owen et al., 2009).

Sin embargo, las principales críticas evidencian que el análisis técnico estándar, en esa caja negra que ha sido la evaluación del riesgo, soporta indeterminaciones técnicas, sociales y éticas difícilmente tolerables. De manera breve y concisa, podemos destacar los siguientes resultados concluyentes, en los Estudios de Ciencia y Tecnología: la caracterización del riesgo incorpora en su seno actividades previamente definidas como no deseadas y adversas; el procedimiento se guía por la determinación normativa de valores metodológicos anteriormente fijados y establecidos; el análisis estándar resulta insuficiente en aquellos sistemas que obligan la toma de decisión bajo incertidumbre, ignorancia e indeterminación; el carácter técnico y aplicativo es indiferente a la distinción entre la gravedad de las probabilidades y la magnitud del daño, algo que por el contrario resulta nuevamente insostenible a nivel social y ético (Adam y Groves, 2011; Mitcham, 2003; Stehr, 2005).

\section{La emergencia de una ciudadanía exigente}

En tercer lugar, es necesario captar la idea de una ciudadanía reflexiva, conocedora de las dinámicas políticas de la ciencia y crecientemente preocupada ante los motivos que guían el cambio tecnológico (Bauer, Shukla y Allum, 2011). Desmentida empíricamente la hipótesis del déficit, que impone una correlación entre el nivel de conocimiento (de la dimensión cognitiva) de la ciencia y la percepción social, los resultados sociológicos distinguen entre la ciencia en abstracto (hay una confianza en la ciencia y la profesión científica) y la ciencia en contexto (Kallerud y Ramberg, 2002).

La desconfianza está ligada a las dimensiones sociales e institucionales de la actividad científico-tecnológica. Entre las variables explicativas se imponen (Eizagirre, 2013b; Leach, Scoones y Wynne, 2005): la competencia cognitiva y social que 
merecen los expertos y asesores; los efectos de segundo orden que provoca la ciencia al representar e intervenir la realidad; la percepción de una convergencia de intereses entre expertos y autoridades; el origen humano del riesgo, su carácter incierto y de magnitud ilimitada, así como la incertidumbre como aspecto endémico del conocimiento; la distorsión deliberada de la información y/o la no transmisión de la información disponible. A todo ello, debemos agregar que ahora los conocimientos se producen fuera del laboratorio y en contextos de aplicación y que la intervención científico-tecnológica puede llegar a tener un carácter global, irreversible, no-asegurable, imprevisible y en todo caso originado por la actividad humana (Hommels, Mesman y Bijker, 2014; Stehr y Grundmann, 2011).

\section{El nuevo contexto de las políticas de I+D}

Aunque sea muy sucintamente, hemos identificado los principales resultados que nos aportan los Estudios de Ciencia y Tecnología, referidos al ámbito social e institucional de la ciencia y su relación con la sociedad, y que sirven para comprender el contexto desafiante en el que se sitúan las políticas de I+D y su relación con la sociedad.

Las políticas de I+D han modificado sus modelos de estímulo, promoción y legitimación de la innovación y han ampliado sus funciones también a la regulación de los efectos adversos de la actividad científico-tecnológica. A este hecho se unen, como hemos indicado, la emergencia de nuevos modos de producir, validar y justificar los conocimientos, así como una ciudadanía más exigente en sus deseos y expectativas (Hessels y van Lente, 2008; Nowotny, Scout y Gibbons, 2001). Esta transformación en la producción, regulación y evaluación del conocimiento sugiere una extensión de las posibilidades operativas.

Ahora bien, frente a la imagen lineal, académica y demarcacionista de las políticas de ciencia, hemos visto que los Estudios de Ciencia y Tecnología tratan de singularizar las nuevas políticas del conocimiento en un registro caracterizado por tres rasgos distintivos. Por una parte, las prácticas científico-tecnológicas están orientadas en contextos en el que interactúan múltiples y heterogéneos agentes (Jasanoff, 2005). Por otra parte, las prácticas científicas y las innovaciones se desarrollan habitualmente en situaciones de controversia, riesgo e incertidumbre (Renn, 2008). En tercer lugar, se evalúan y validan crecientemente según criterios de calidad y relevancia exógenos a los de las disciplinas y comunidades científicas (Lentsch y Weingart, 2011; Pielke, 2007; Stirling, 2007).

Se trata de un hecho singular y en parte paradójico: en un contexto socio-histórico regido de manera creciente a través del conocimiento y que institucionaliza mecanismos reflexivos y tipos de acción propios de la investigación científica en todos los ámbitos funcionales de la vida social e institucional, también se cuestiona la imagen de la ciencia que al amparo del conocimiento teórico asocia automáticamente el conocimiento seguro, la innovación científico-técnica y el progreso social (Jasanoff, 2012; Stirling, 2008). Cuando más necesitamos el conocimiento, mayor es también su vulnerabilidad, y mayor es a la vez, su instrumentalización por una diversidad de intereses concurrentes. 
De ahí concluimos que la gobernanza de la ciencia debe promover y garantizar el conocimiento robusto, pertinente y relevante en los procesos de decisión política (Pereira y Funtowicz, 2009; Grande et al., 2013). Es a nuestro parecer el reto que integra en su seno la nueva propuesta ligada al enfoque IIR.

\section{El enfoque IIR en contexto}

El enfoque IIR aboga por comprender la gobernanza de la ciencia y la innovación como un principio democratizador que promueve una interacción entre agentes diversos que integran valores, preocupaciones, intenciones y propósitos heterogéneos. En este sentido, su propósito de integrar en el proceso mismo de generación y producción del conocimiento científico-tecnológico las distintas sensibilidades y los intereses concurrentes, puede suponer un punto de inflexión. ${ }^{2}$

El punto de partida radica en la aparente paradoja del nuevo contexto social de la actividad científico-tecnológica sometida, por un lado, a una creciente demanda de modelos responsables de investigación y, por otro, instrumentalizada para resultados macroeconómicos. Otra paradoja adicional se debe a que la ciencia se concibe como una fuente de riesgos ambientales, socioéticos y sanitarios al tiempo que se valora su rol esencial en la gobernanza de esos riesgos.

En este contexto, los sistemas de ciencia, tecnología e innovación se constituyen en la complejidad que actitudes, estrategias, preferencias, capacidades y prospecciones heterogéneas configuran. La nueva modalidad de actividad científica se constituye en ese encadenamiento de interacciones y de operaciones que mediaciones interactivas entre objetos naturales, técnicos y sociales producen, validan y justifican. Siendo esto así, nuestra interpretación de un enfoque IIR, apunta que, ese juego de interacciones entre las dinámicas de la ciencia y de la sociedad obliga modelos de gobernanza que coordinen posiciones generalmente concurrentes y contradictorios, a la vez que se alinean con trayectorias de investigación e innovación responsables (Special Eurobarometer, 2013).

La integración de enfoques IIR puede interpretarse como una respuesta institucional a la experiencia de que innovaciones económicamente viables pueden fracasar al ser cuestionadas socialmente. La experiencia analítica y práctica adquirida en las diferentes naciones europeas también exige revisar definitivamente los modelos de gobernar la ciencia y la tecnología. Ahora bien, nos parece prematuro sugerir que la democracia y la reflexividad también se extienden a la ciencia y la

2 La introducción del concepto de investigación e innovación responsables (IIR) se presenta como un elemento clave del 8. Programa Marco de I+D de la Unión Europea, "Horizonte 2020". En este sentido, según el Parlamento y Consejo Europeos, "Horizon 2020 should favour an informed engagement of citizens and civil society on research and innovation matters [...] by developing responsible research and innovation agendas that meet citizens' and civil society's concerns and expectations and by facilitating their participation in Horizon 2020 activities" (pp. 8-9). [Proposal for a regulation of the European Parliament and of the Council establishing Horizon 2020 - The Framework Programme for Research and Innovation (2014-2020), Brussels, 30/11/2011, COM(2011) 809 final, 2011/0401 (COD)]. 
tecnología. Por el contrario, en este artículo planteamos que una comprensión integral de la integración de enfoques IIR requiere capturar el modo en que la responsabilidad aplicada al desarrollo tecno-económico confronta los intereses económicos y políticos que inducen y contextualizan las políticas de ciencia y tecnología.

La nueva retórica no puede descuidar las dinámicas científico-técnicas, políticas, económicas y sociales por las cuales se aplican (y se dejan de aplicar) enfoques de tipo IIR en los diseños de las agendas de I+D. De esta manera, aquí sugerimos que el significado, alcance y aplicación de un enfoque IIR dependerá de las dinámicas económicas y sociopolíticas por las cuales se instrumentalizan y regulan la ciencia y la tecnología. En otras palabras, el modo como representamos y enmarcamos la gobernanza de la investigación y la innovación decidirá muchos problemas epistemológicos y normativos, la calidad del espacio público así como nuestras acciones posibles y deseables. En el apartado anterior hemos sugerido algunos resultados, en ese sentido. Se trata de un asunto que se alimenta simultáneamente por nuevos horizontes, narrativas sociotécnicas alternativas y viejos problemas irresueltos.

Frente a las concepciones tradicionales de gobierno en el registro de la visión lineal, determinista y triunfalista que presupone la causalidad o en el más reciente del paradigma Ciencia y Sociedad que asume todavía los criterios demarcacionistas y en el que los resultados están ligados a la maximización económica y los impactos se reducen a riesgos técnicos calculables; el significado, alcance y aplicación de un enfoque IIR que están incorporando en la Unión Europea las políticas comunitarias y nacionales, dependerá de las dinámicas económicas y sociopolíticas por las cuales se instrumentalizan y regulan la ciencia y la tecnología.

\section{El significado y alcance de la IIR}

Una tarea a la que someterse es la discusión sobre el significado, alcance y aplicación de un enfoque IIR. En esa dirección, creemos interesante plantear tres ejes que, desde los Estudios de Ciencia y Tecnología, contribuirán a definir y evaluar el significado y alcance de un enfoque IIR en las políticas de I+D comunitarias y nacionales. La tarea debe comprometerse en el diseño de modelos de gobernanza en perspectiva de IIR que faciliten la evaluación y transformación de las políticas de I+D. En otras palabras, debe evaluar y promover las dinámicas anticipatorias, reflexivas, deliberativas y responsables que debe integrar un enfoque de IIR realmente transformador. Como puede apreciarse, aquí proponemos que los Estudios de Ciencia y Tecnología deben simultáneamente evaluar la implementación de un enfoque IIR, a la vez que promueven su diseño en colaboración con otros agentes involucrados en los procesos de investigación e innovación.

Un primer eje de análisis debe centrarse en el carácter multidimensional de la política científica en su doble función de promoción y regulación de la actividad científico-tecnológica. Se está consolidando una nueva modalidad caracterizada por estar orientada en contextos de aplicación, guiada por disciplinas y 
conocimientos plurales, validada por criterios de calidad exógenos a los de las disciplinas, y justificada por intereses socioeconómicos.

En este nuevo registro de producción, validación y evaluación, el juego de interacciones entre las dinámicas de la ciencia y de la sociedad se constituye actualmente en la negociación entre la creciente instrumentalización de la ciencia y las mayores exigencias sociales ligadas a la integridad de los investigadores y su responsabilidad ante las implicaciones éticas, legales y sociales (Guston y Sarewitz, 2006; Levidow y Carr, 2010; Macnaghten, 2010). Estas prácticas científico-tecnológicas ambivalentes guardan poca relación con la imagen positivista que presupone una correlación entre validez del conocimiento y su aislamiento de consideraciones no científicas (Hoppe, 1999; Wilsdon, Wynne y Stilgoe, 2005). El seguimiento de aquellas prácticas de investigación responsables indica un primer eje para valorar el alcance de un enfoque IIR.

En este sentido, los Estudios de Ciencia y Tecnología se convierten así propicios para entender lo que son las prácticas científicas. Su principal contribución se debe a sus análisis de los mecanismos que operan en la producción del conocimiento (Stehr y Meja, 2005). Así, los análisis permiten cuestionar los supuestos, valores y coaliciones que guían la ciencia y los tipos del saber, proponer una nueva actividad pública de la ciencia, generar nuevas redes epistémicas que integran capacidades de producción de conocimiento diferentes, y afrontar nuevos espacios de intersección de lo científico y lo político.

En todo caso, la tarea se compromete con la siguiente cuestión: ¿cómo diseñar políticas que aspiren a institucionalizar este debilitamiento de la autoridad epistémica de la ciencia, pero coincidente con la valorización de la investigación y la innovación como motor de la competitividad económica, junto con la incorporación de las dimensiones ambientales, éticas y sociales en la constitución del conocimiento validado?

Un segundo eje tiene que evaluar la institucionalización de los mecanismos de reflexividad, anticipación e inclusión en los procesos de investigación e innovación. Encontramos antecedentes recientes en las iniciativas europeas sobre ciencia y sociedad (EC, 2001; 2002), que promueve una ciencia responsable como núcleo de las políticas, y se pregunta por las dimensiones éticas de la actividad científico-tecnológica, por la gobernanza en materia de riesgos y por la utilización social de los conocimientos. También se debe señalar la integración sociotécnica que desde el Quinto Programa Marco Europeo se promueve a través de la colaboración entre ciencias sociales, naturales y físicas.

No obstante, en un enfoque IIR, la validación responsable de los procesos de investigación que integran las consideraciones éticas, sociales y ambientales debe desafiar el modelo lineal y la regulación basada en el riesgo como paradigma dominante de la gobernanza de la ciencia y la innovación (Mitcham, 2003; Pellizzoni, 2004; Stephanie y Fisher, 2011). Este paradigma se reconoce por sus limitaciones en la gestión que anticipan las áreas emergentes como son entre otros los organismos genéticamente modificados, la biología sintética, la tecnología nuclear, la geoingeniería, las nanotecnologías, y las tecnologías de la información y la comunicación (Kastenhofer, 2011). 
La institucionalización de la responsabilidad sugiere la relevancia que adquiere la anticipación como descripción y análisis de los impactos económicos, sociales y ambientales no deseados e incluso no previstos (Christensen et al., 2012; den Boer, Rip y Speller, 2009). Esto precisa de metodologías basadas en la evaluación de las tecnologías, el principio de precaución, la gobernanza anticipatoria y el desarrollo de escenarios (Eizagirre, 2011; Hellstrom, 2003; Rip, Misa y Schot, 1995; van den Hoven, Lokhorst y van del Poel, 2012; von Schomberg, 2011; 2012). Frente a modelos lineales, deterministas y triunfalistas, la integración sociotécnica se define por la apertura y las alternativas, por reflexionar sobre objetivos, motivos e impactos conocidos y no conocidos, y por estimular estas consideraciones a través de deliberaciones colectivas (Kaiser, 2010). Los Estudios de Ciencia y Tecnología han participado en la construcción de estos distintos principios y metodologías de participación, pero ahora deben ampliar su tarea a los ámbitos en los que realmente se generan y producen las innovaciones científico-tecnológicas.

De hecho, en los últimos años han proliferado actividades - heterogéneas en su naturaleza y objetivos - ligadas a la participación social, que sin embargo han suscitado críticas en las ciencias sociales por su carácter instrumental, legitimador y superficial (Irwin, Jensen y Jones, 2013; Wilsdon y Willis, 2004; Wynne, 2006). A partir de estas críticas, un enfoque IIR se pregunta: ¿cómo formalizar procesos de investigación e innovación responsables, instigados desde su inicio en la definición y luego en la progresión, dirección, deliberación y legitimación, por la multidisciplina y atenta también a las ciencias sociales y humanas?

El significado y alcance de un enfoque IIR, tiene como tercer eje, una dimensión normativa que, en nuestras sociedades democráticas y pluralistas, va a generar nuevas controversias. Desde una postura a-crítica, puede sugerirse la necesidad de posibilitar una investigación e innovación responsables que capacite para responder a los retos sociales de futuro que imponen los cambios de tendencia en la sociedad global (Maasen y Weingart, 2005; Fisher, Mahajan y Mitcham, 2006; Groves, 2006). El gobierno de la ciencia no puede centrarse en mitigar los efectos no deseados de la innovación, que definidos como riesgos no intencionados, establece mecanismos de regulación en el eslabón último de la innovación. De la misma manera, el programa "Ciencia en Sociedad" de la Comisión Europea y la emergencia de la IIR en los discursos políticos de la Unión Europea, sugieren una preocupación ligada a los objetivos, motivaciones e intenciones (Owen, Macnaghten y Stilgoe, 2012; von Schomberg y Davies, 2010).

Ahora bien, esos ingredientes indican la necesidad de discutir también sobre el tipo de ciencia que necesita una sociedad, lo que involucra una postura crítica, de tal modo que la apertura y la deliberación validen el proceso multidireccional de variación y selección que consolida las formas concretas de tecnología (Stilgoe y Lock, 2014; Stirling, 2011). Frente al modelo "Ciencia y Sociedad" para el que la ampliación se entiende como la revisión y aprobación ética de las conductas de investigación que envuelven personas, animales y material genético, por el contrario el discurso de la IIR propone democratizar la gobernanza de los objetivos a perseguir en ciencia y tecnología (Owen, Bessant y Heintz, 2013). Es lo que nos permite discutir sobre el tipo de ciencia que necesita una sociedad. 
Aquí surgen las primeras controversias. Una respuesta pragmática - como propone von Schomberg, director general de Investigación e Innovación de la Comisión Europea - se encuentra en los valores consagrados en la Constitución Europea y en los objetivos de la estrategia "Horizonte 2020". No obstante, se trata de una discusión inherentemente política e integra aspectos vinculados al poder, la dominación y el conflicto (Jacob, 2013). En definitiva, el tercer eje de análisis y evaluación, comprometido con el enfoque IIR, se plantea la siguiente cuestión: ¿cuáles son los impactos adecuados y justos de la actividad científico-tecnológica y sobre qué valores deben anclarse?

Estos ejes y su tratamiento muestran que, frente a las concepciones tradicionales sobre ciencia, tecnología, políticas públicas y sociedad, es razonable plantear que la gobernanza decide la extensión de las posibilidades operativas y por ello debe promoverse una organización de la coproducción del conocimiento robusta, justa y relevante. En ese juego se dilucidará si la IIR justifica una transformación superficial, o si por el contrario se convierte en el dispositivo que impulsa una transformación estructural de las relaciones entre ciencia, tecnología y sociedad. En este apartado hemos querido avanzar algunas pistas para su respuesta, a la vez que hemos sugerido el rol que los Estudios de Ciencia y Tecnología tienen por delante.

\section{Consideraciones finales}

El enfoque IIR se propone como finalidad abordar el problema de la gobernanza de la ciencia en los nuevos contextos de estímulo, producción, regulación y valoración de la ciencia. Este artículo ha propuesto un diálogo entre el nuevo lenguaje sobre las políticas de I+D y los Estudios de Ciencia y Tecnología.

Hay una creciente aproximación entre el modelo "Ciencia en Sociedad" y los resultados que han generado y validado los Estudios de Ciencia y Tecnología. En diversas ocasiones, los protagonistas de ambos campos (el campo político y el campo académico) han interactuado mutuamente. De ahí que, en cierta medida, buena parte de las tareas que integra el enfoque IIR, los interpele simultáneamente.

En un contexto que para acreditar una decisión debe recurrirse al conocimiento pero su recurso no garantiza consenso en su validación ni aceptabilidad social, ¿cómo diseñar mecanismos de construcción cognitivas e institucionales mutuas? ¿Cómo validar decisiones que requieren representación democrática y racionalidad científica pero en contextos que se caracterizan por la incertidumbre y el pluralismo, por expectativas cognitivas y normativas contrapuestas, por decisiones socioambientales únicas e irreversibles? En un contexto que la verdad no puede administrar el gobierno, la gobernanza significa diseñar el ensamblaje de la democracia con modelos de investigación e innovación responsables. Para ello resultan centrales estas cuestiones: ¿cómo trazar y caracterizar las fronteras cognitivas, normativas e institucionales en la producción del conocimiento? ¿Cómo validar competentes las autoridades ("la experticia") cuando la robustez y la relevancia del conocimiento transgreden su naturaleza $\mathrm{y}$ fronteras? 
La tarea amplía y transforma los marcos tradicionales de atención en el estudio de la gestión de la ciencia. En él, las cuestiones planteadas se traducen en la administración de la interacción de dos procesos separados ("experticia" y "poder"). El enfoque IIR desplaza su análisis y su gestión al modo como se incrustan el conocimiento del mundo natural y la acción política y se co-constituyen. La tarea se interesa por contextos de gobernanza específicos en que se constituyen mutuamente el orden natural y social. Esta precisión analítica requiere de perspectivas y metodologías que faculten la integración de elementos heterogéneos (materiales, cognitivos, organizacionales), de aspectos vinculados al poder y el conflicto, mediadores en la representación, generación y transmisión del conocimiento.

Ello se debe a que la actividad científico-tecnológica (ciencia como acción) tiene un carácter multidimensional en el que interactúan agentes heterogéneos con intereses diversos en la producción, validación y legitimación del proceso de innovación. En segundo lugar, las tecnologías emergentes incorporan un conjunto amplio de dimensiones naturales, sociales y humanas, y el enfoque IIR se propone reflexionar sobre los aspectos éticos, la apropiación de la tecnología y el desarrollo económico, la gobernanza, la evaluación de las tecnologías, la sostenibilidad, la integración sociotécnica, etc.

Las tareas indicadas también configuran una combinación de estrategias orientadas a describir las interrelaciones entre elementos que construyen la red sociotécnica; comprender el rol de los distintos agentes y su influencia en la estructuración de la política de I+D; proponer marcos de análisis y evaluación orientadas a mejorar las redes sociotécnicas en base a mecanismos de gobernanza responsables y socialmente deseables. Cada uno de los conocimientos generados resulta además en beneficio de futuras tareas como diseñar e implantar modelos para monitorizar las políticas de I+D y su cultura científica desde la perspectiva "Ciencia en Sociedad".

El significado y alcance de un enfoque IIR está relacionado a la generación de nuevas redes epistémicas y sociotécnicas que integran capacidades de producción de conocimiento en respuesta a investigaciones e innovaciones responsables y socialmente deseables. En definitiva, los principios normativos y los valores de un enfoque IIR no deben entenderse como dados de antemano. Por el contrario, sus significados y su alcance dependerán de las dinámicas que instrumentalizan y regulan las políticas de I+D. Así, por nuestra parte, reconocemos la heurística política de una perspectiva de IIR, a la vez que sugerimos la necesidad de revisar los supuestos que estructuran los modelos de gobernanza y los ideales normativos de la ciencia que liberan a las políticas diseñadas y sus objetivos del escrutinio público. Es lo que realmente permitirá un cambio sustancial en las agendas políticas y promoverá la discusión sobre el tipo de ciencia y tecnología que consideramos justo, adecuado y deseable.

\section{Bibliografía}

Adam, Barbara, y Chris Groves (2011), “Futures tended: care and future-oriented responsibility", Bulletin of Science, Technology \& Society, 31, pp. 17-27. 
Adger, Neil, y Andrew Jordan (orgs.) (2009), Governing Sustainability, Cambridge, UK, Cambridge University Press.

Anichini, Giulia, y Suzanne de Cheveigné (orgs.) (2012), "Science in society in Europe", Science and Public Policy, 39 (6), número especial, pp. 701-760.

Barben, Daniel (2007), “Changing regimes of science and politics: comparative and transnational perspectives for a world in transition", Science and Public Policy, 34 (1), pp. 55-69.

Bauer, Martin, Rajesh Shukla, y Nick Allum (orgs.) (2011), The Culture of Science. How Does the Public Relate to Science Across the Globe, Londres, Routledge.

Berkhout, Frans, Melissa Leach, e Ian Scoones (2004), Negotiating Environmental Change. New Perspectives from Social Science, Cheltenham, UK, Edward Elgar.

Bolay, Jean-Claude, Magali Schmid, Gabriela Tejada, y Eileen Hazboun (orgs.) (2012), Technologies and Innovations for Development. Scientific Cooperation for a Sustainable Future, París, Springer-Verlag.

Bozeman, Barry, y Daniel Sarewitz (2011), "Public value mapping and science policy evaluation", Minerva, 49 (1), pp. 1-23.

Callon, Michel, Pierre Lascoumes, y Yannick Barthe (2010), Acting in an Uncertain World. An Essay on Technical Democracy, Cambridge, MA, The MIT Press.

Canaan, Joyce, y Wesley Shumar (orgs.) (2008), Structure and Agency in the Neoliberal University, Nueva York, Routledge.

Christensen, Steen Hyldgaard, Carl Mitcham, Bocong Li, y Yanming An (orgs.) (2012), Engineering, Development, and Philosophy. American, Chinese, and European Perspectives, Nueva York, Springer-Verlag.

Den Boer, Duncan, Ari Rip, y Sylvia Speller (2009), "Scripting posible futures of nanotechnologies: a methodology that enhances reflexivity", Technology in Society, 31, pp. 295-304.

EC - European Commission (2001), European Governance. A White Paper, Bruselas, EC.

EC - European Commission (2002), Science and Society. Action Plan, Luxemburgo, EC.

Echeverría, Javier (2002), Ciencia y Valores, México, DF, Fondo de Cultura Económica.

Eizagirre, Andoni (2009), "Las nuevas iniciativas europeas sobre ciencia y sociedad", Sistema, 210, pp. 3-22.

Eizagirre, Andoni (2011), "La precaución como principio de acción sostenible”, Isegoría, Revista de Filosofía Moral y Política, 44, pp. 303-324.

Eizagirre, Andoni (2013a), “Gobernar el conocimiento", Isegoría, Revista de Filosofía Moral y Política, 48, pp. 229-244.

Eizagirre, Andoni (2013b), “Las percepciones sociales en Europa sobre el rol de la ciencia y la tecnología", Revista de Estudios Sociales, 48, pp. 67-78.

Eizagirre, Andoni (2015), "El discurso sobre las políticas de ciencia y tecnología”, Argumentos de Razón Técnica, 18, pp. 39-68.

Felt, Ulrich (reportero) (2007), Science and Governance. Taking European Knowledge Society Seriously, Bruselas, Report of the Expert Group on Science and Governance to DG Research.

Fisher, Erik, Roop L. Mahajan, y Carl Mitcham (2006), “Midstream modulation of technology: governance from within", Bulletin of Science, Technology E Society, 26 (6), pp. 485-496. 
Godin, Benoit (2010), The Making of Science, Technology and Innovation Policy. Conceptual Frameworks as Narratives, 1945-2005, Montreal, Centre Urbanisation Culture Société.

Grande, Edgar, Dorothea Cansen, Otfried Jarren, Arie Rip, Uwe Schimank, y Peter Weingart (orgs.) (2013), Neue Governance der Wissenschaft. Reorganisation - Externe Anforderungen - Medialisierung, Bielefeld, Transcript.

Groves, Chris (2006), "Technological futures and non-reciprocal responsibility”, The International Journal of Humanities, 4 (2), pp. 57-61.

Guston, David, y Daniel Sarewitz (2006), Shaping Science and Technology Policy. The Next Generation of Research, Madison, WI, The University of Wisconsin Press.

Hellstrom, Tomas (2003), "Systemic innovation and risk: technology assessment and the challenge of responsible innovation", Technology in Society, 25, pp. 369-384.

Hessels, Lauren, y Harron van Lente (2008), "Re-thinking new knowledge production: a literature review and a research agenda", Research Policy, 37 (4), pp. 740-760.

Hommels, Anique, Jessicca Mesman, y Wiebe Bijker (orgs.) (2014), Vulnerability in Technological Cultures. New Directions in Research and Governance, Cambridge, MA, The MIT Press.

Hoppe, Robert (1999), "Policy analysis, science and politics: from 'speaking truth to power' to 'making sense together' ", Science and Public Policy, 26 (3), pp. 201-210.

Irwin, Alan (2006), "The politics of talk: coming to terms with the 'New' Scientific Governance", Social Studies of Science, 36 (2), pp. 299-320.

Irwin, Alan (2008), "STS perspectives on scientific governance", en Edward Hackett, Olga Amsterdamska, Michael Lynch y Judy Wajcman (orgs.), The New Handbook of Science and Technology Studies, Cambridge, MA, The MIT Press, pp. 583-607.

Irwin, Alan, Tolber Elgaard Jensen, y Kevin Jones (2013), "The good, the bad and the perfect: criticizing engagement practice", Social Studies of Science, 43 (1), pp. 119-136.

Jacob, Klaus (reportero) (2013), Options for Strengthening Responsible Research and Innovation. Report of the Expert Group on the State of Art in Europe on Responsible Research and Innovation, Bruselas, Directorate General for Research and Innovation.

Jamison, Andrew (2012), "Science and technology in postwar Europe", en Dan Stone (org.), The Oxford Handbook of Postwar European History, Oxford, Oxford University Press, pp. 630-648.

Jasanoff, Sheila (org.) (2004), States of Knowledge. The Co-Production of Science and Social Order, Londres, Routledge.

Jasanoff, Sheila (2005), Designs on Nature. Science and Democracy in Europe and the United States, Princeton, NJ, Princeton University Press.

Jasanoff, Sheila (2012), Science and Public Reason, Nueva York, Routledge.

Joly, Pierre-Benoit, Claire Marris, y Olivier Marcant (2001), La Constitution d'Un "Problème Public". La Controverse sur les OGM et Ses Incidences sur la Politique aux Etats-Units, Grenoble, Institut National de la Recherche Agronomique.

Kaiser, Mario (org.) (2010), Governing Future Technologies. Nanotechnology and the Rise of an Assessment Regime, Dordrecht, Springer-Verlag.

Kallerud, Egil, e Inge Ramberg (2002), "The order of discourse in surveys of public understanding of science", Public Understanding of Science , 11 (3), pp. 213-224. 
Kastenhofer, Karen (2011), “Risk assessment of emerging technologies and post-normal science", Science, Technology \& Human Values, 36 (3), pp. 307-333.

Kleinman, Daniel Lee, y Steven Vallas (2001), “Science, capitalism, and the rise of the 'knowledge worker': the changing structure of knowledge production in the United States", Theory and Society, 30 (4), pp. 451-492.

Lane, Stuart, Francisco Klauser, y Mathew Kearnes (2012), Critical Risk Research. Practices, Politics and Ethics, Oxford, Wiley-Blackwell.

Leach, Melissa, Ian Scoones, y Brian Wynne (orgs.) (2005), Science and Citizens. Globalization and the Challenge of Engagement, Londres, Zed Books.

Leach, Melissa, Ian Scoones, y Andy Stirling (2010), Dynamic Sustainabilities. Technology, Environment, Social Justice, Londres, Zed Books.

Lentchs, Justus, y Peter Weingart (orgs.) (2011), The Politics of Scientific Advice. Institucional Design for Quality Assurance, Cambridge, UK, Cambridge University Press.

Levidow, Les, y Susan Carr (2010), GM Food on Trial. Testing European Democracy, Londres, Routledge.

Liberatore, Angela (2001), Democratising Expertise and Establishing Scientific Reference Systems, Bruselas, European Commission.

Maasen, Sabine, y Peter Weingart (orgs.) (2005), Democratization of Expertise? Sociology of the Sciences Yearbook, Dordrecht, Springer-Verlag.

Macnaghten, Paul (2010), "Reserching technoscientific concerns in the making", Environment and Planning A, 41, pp. 23-37.

Marklund, Göran, Nicholas S. Vonortas, y Charles W. Wessner (orgs.) (2009), The Innovative Imperative. National Innovation Strategies in the Global Economy. Cheltenham, UK, Edward Elgar.

Miettinen, Reijo (2013), Innovation, Human Capabilities, and Democracy. Towards an Enabling Welfare State, Oxford, Oxford University Press.

Mirowski, Philip, y Esther-Mirjam Sent (2008), "The commercialization of science and the response to STS", en Edward Hackett, Olga Amsterdamska, Michael Lynch y Judy Wajcman (orgs.), The Handbook of Science and Technology Studies, Cambridge, MA, The MIT Press, pp. 635-689.

Mitcham, Carl (2003), "Co-responsibility for research integrity”, Science and Engineering Ethics, 9, pp. 273-290.

Mitcham, Carl (org.) (2005), Encyclopedia of Science, Technology, and Ethics, Nueva York, Macmillan Reference.

Nowotny, Helga, Peter Scout, y Michael Gibbons (2001), Re-thinking Science. Knowledge and the Public in an Age of Uncertainty, Cambridge, UK, Polity Press.

OECD (2010), The OECD Innovation Strategy. Getting a Head Start on Tomorrow, París, OECD.

OECD (2012), Meeting Global Challenges through Better Governance, París, OECD.

Pellizzoni, Luigi (2004), "Responsibility and environmental governance", Environmental Politics, 13, pp. 541-565.

Owen, Richard, David Baxter, Trevor Maynard, y Michael Depledge (2009), “Beyond regulation: risk princing and responsible innovation", Environmental Science $\mathcal{E}$ Technology, 43, pp. 5171-5175. 
Owen, Richard, Paul Macnaghten, y Jack Stilgoe (2012), “Responsible research and innovation: from science in society to science for society, with society", Science and Public Policy, 39, pp. 751-760.

Owen, Richard, John Bessant, y Maggy Heintz (2013), Responsible Innovation. Managing the Responsible Emergence of Science and Innovation in Society, Chichester, Wiley.

Ozolina, Zaneta, Carl Mitcham, Doris Shroeder, Emilio Mordini, Paul McCarthy, y John Crowley (2012), Ethical and Regulatory Challenges to Science and Research Policy at Global Level, Luxemburgo, Publication Office of the European Union.

Pereira, Angela-Guimaraes, y Silvio Funtowicz (2009), Science for Policy. New Challenges, New Opportunities, Oxford, Oxford University Press.

Pielke, Roger (2007), The Honest Broker. Making Sense of Science in Policy and Politics, Cambridge, UK, Cambridge University Press.

Renn, Ortwin (2008), Risk Governance. Coping with Uncertainty in a Complex World, Oxford, Rowman \& Littlefield Publishers.

Rip, Arie, Thomas Misa, y Johan Shot (orgs.) (1995), Managing Technology in Society. The Approach of Constructive Technology Assessment, Londres, Thomson.

Shrader-Frechette, Kristin (1985), Risk Analysis and Scientific Method. Methodological and Ethical Problems with Evaluating Societal Hazards, Boston, Kluwer.

Shrader-Frechette, Kristin (1991), Risk and Rationality, Berkeley, CA, University of California Press.

Shrader-Frechette, Kristin (2011), Taking Action, Saving Lives. Our Duties to Protect Environmental and Public Health, Oxford, Oxford University Press.

Slaughter, Sheila, y Gary Rhoades (2004), Academic Capitalism and the New Economy. Markets, State, and Higher Education, Baltimore, MD, The Johns Hopkins University Press.

Special Eurobarometer 401 (2013), Responsible Research and Innovation (RRI), Science and Technology, Bruselas, European Commission.

Stehr , Nico (org.) (2005), Knowledge Politics. Governing the Consequences of Science and Technology, Boulder, CO, Paradigm Publishers.

Stehr, Nico, y Voker Meja (orgs.) (2005), Society E Knowledge. Contemporary Perspectives in the Sociology of Knowledge E Science, New Brunswick, NJ, Transaction Publishers (2. ․ edición revisada).

Stehr, Nico, y Reiner Grundmann (orgs.) (2011), Experts. The Knowledge and Power of Expertise (Key Ideas), Nueva York, Routledge.

Stephanie, Bird, y Erik Fisher (orgs.) (2011), "Science and technology policy in the making: observation and engagement", Science and Engineering Ethics, 17 (4), número especial, pp. 607-849.

Stilgoe, Jack, y Simon Lock (2014), “Why should we promote public engagement with science?", Public Understanding of Science, 23 (1), pp. 4-15.

Stirling, Andrew (2007), “Deliberative futures: precaution and progress in social choice of sustainable technology", Sustainable Development, 15 (5), pp. 286-295.

Stirling, Andrew (2008), “Opening up or closing down? Power, participation, and pluralism in the social appraisal of technology", Science Technology Human Values, 33 (2), pp. 262-294. 
Stirling, Andrew (2011), "Pluralising progress: from integrative transitions to transformative diversity", Journal of Environmental Innovation $\mathcal{E}$ Societal Transitions, 1 (1), pp. 82-88.

van den Hove, Sylbille, Jacqueline McGlade, Pierre Mottet, y Michael Depledge (2012), "The innovation union: a perfect means to confused ends?", Environmental Science and Policy, 16 (1), pp. 73-80.

van den Hoven, Jeroen, Gert-Jan Lokhorst, e Ibo van den Poel (2012), “Engineering and the problem of moral overload", Science and Engineering Ethics, 18, pp. 1-13.

von Schomberg, René (org.) (2011), Towards Responsible Research and Innovation in the Information and Communication Technologies and Security Technologies Fields, Luxemburgo, Publication Office of the European Union.

von Schomberg, René (2012), “Prospects for technology assessment in a framework of responsible research and innovation", en Marc Dusseldorp y Richard Beecroft (orgs.), Technikfolgen abschätzen lehren. Bildungspotenziale transdisziplinärer Methoden, Dordrecht, Springer-Verlag, pp. 39-61.

von Schomberg, René, y Sarah Davies (orgs.) (2010), Understanding Public Debate on Nanotechnology. Options for Framing Public Policy, Luxemburgo, Publication Office of the European Union.

Wilsdon, James, y Rebecca Willis (2004), Seethrough Science. Why Public Engagement Needs to Move Upstream, Londres, Demos.

Wilsdon, James, Brian Wynne, y Jack Stilgoe (2005), The Public Value of Science. Or How to Ensure that Science Really Matters, Londres, Demos.

Wynne, Brian (2002), "Risk and environmental as legitimatory discourses of technology: reflexivity inside out?", Current Sociology, 50 (3), pp. 459-477.

Wynne, Brian (2006), "Public engagement as a means of restoring public trust in science - hitting the notes, but missing the music", Community Genetics, 9, pp. 211-220.

Andoni Eizagirre. Profesor titular de Mondragon Unibertsitatea, Facultad de Humanidades y Ciencias de la Educación, Goiru Kalea, 20500 Arrasate, Gipuzkoa, España. E-mail: aeizagirre@mondragon.edu

Receção: 1 de agosto de 2014 Avaliação: 25 de março de 2016 